\section{1) Membrane protein structures}

New methods addressing the challenges in membrane protein expression, solubilization and crystallization promise to yield many more atomic structures.

Membrane proteins make up roughly $30 \%$ of the proteome, but they are highly underrepresented among atomic structures solved, making up less than $1 \%$ of the structures in the Protein Data Bank (PDB). This is not due to a lack of biological importance-indeed, membrane protein receptors make up the bulk of drug targets-but to the technical challenges associated with expressing them in large enough quantities for structural analysis, solubilizing them in appropriate detergents and crystallizing them for structure determination. Even structural genomics efforts, where the goal is to solve representative structures for every protein family, have primarily focused on the

\section{\Optical imaging in thick samples}

Optical methods to image deep into thick samples make it increasingly possible to watch biological processes in vivo.

Biological systems are three-dimensional. Cells are rarely present as isolated monolayers, as in the Petri dish. However, there are technical challenges inherent to in vivo studies, and this is particularly true for imaging.

Biological tissue is opaque and scatters light effectively, and image quality deteriorates rapidly in thicker samples. Conventional confocal microscopy, for instance, cannot image deeper than a few hundred microns into a sample; a fruitfly larva, a mammalian embryo or a tissue slice may be several times thicker. Also, it is often desirable to image such samples in toto, to obtain a global view of tissue organization and gene expression; this too is challenging with conventional approaches. And although

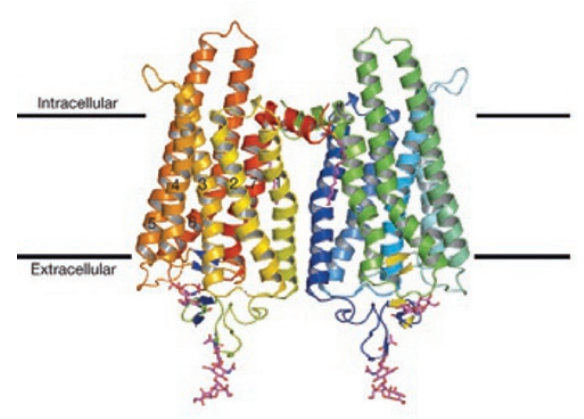

Recently solved crystal structure of the GPCR opsin (Nature 454, 183-187; 2008).

'low-hanging fruit' rather than on more challenging structures such as membrane proteins.

But this is changing as both traditional structural biologists and structural genomics researchers are developing new methods to address the expression, solubilization and crystallization bottlenecks. We are already beginning to see some of the results of these increased efforts. Several landmark papers in 2007 and 2008 reported crystal structures of $G$ protein-coupled receptors (GPCRs), which have been particularly

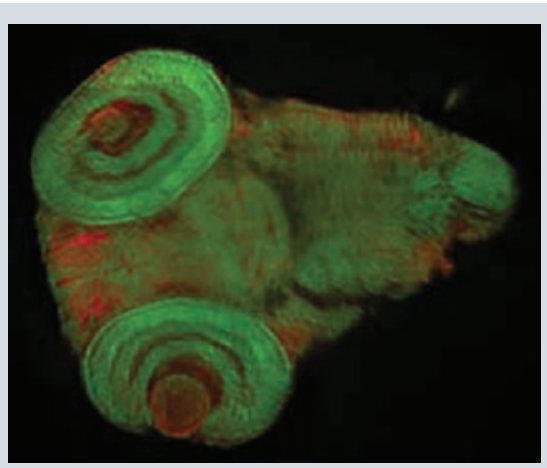

Optical slice through a Medaka embryo (Nat. Methods 4, 311-313; 2007).

techniques such as magnetic resonance imaging (MRI) can image deep into biological samples, the images are low in resolution.

A gap therefore exists between highresolution methods such as confocal microscopy and low-resolution, in vivo methods such as MRI. In recent years, several optical approaches have begun to fill this gap.

Light sheet microscopy, a classical method recently updated, can image a fluorescent signal at a depth of a few millimeters within a sample and has been intractable to structure determination. Structural genomics groups such as NYCOMPS (New York Consortium on Membrane Protein Structures) and CSMP (Center for Structures of Membrane Proteins) in the US, as well as several international initiatives, are setting up high-throughput pipelines to streamline the determination of membrane protein structures, though so far these efforts have resulted in only a handful of new structures. Meanwhile, solid-state NMR methods, which avoid the requirement for crystallization, are rapidly advancing, but sensitivity and resolution enhancements are still needed to make this a robust technology.

There is unlikely to be a 'one-size-fits-all' protocol for obtaining membrane protein structures; rather, a series of methods are needed to expand the variety of proteins accessible to structure determination. Over the next few years, we should expect to see many more methodological solutions for handling membrane proteins, as well as many more membrane protein structures in the PDB.

Allison Doerr

usefully applied to small organisms and embryos. In the past year, a modified version of the approach was used to comprehensively image the cells, numbering in the thousands, in a zebrafish embryo during 24 hours of development (Science 322, 1065-1069; 2008).

Tomographic methods such as optical projection tomography (OPT), by contrast, rely on imaging the sample from many different angles and then using mathematical models to recalculate the original 3D information. OPT can image deeper into a sample (up to $10 \mathrm{~mm}$ ), and it has been combined this past year with in vitro organ culture to image tissue movements and gene expression in the developing mouse limb bud (Nat. Methods 5, 609-612; 2008).

Although these and other volumetric approaches have yet to be taken up widely, this could change as the methods improve and as their capabilities become more commonly appreciated. The possibility of peering deep into organisms and watching biological processes unfold in 3D should be strong motivation. Natalie de Souza 